\title{
Facteurs de variation de la qualité de la fourrure et de la productivité pondérale chez le lapin domestique
}

\author{
H. de ROCHAMBEAU et J.L. VRILLON * \\ I.N.R.A., Station d'Amélioration génétique des Animaux \\ B.P. 27, F 31326 Castanet-Tolosan Cedex \\ * I.N.R.A., Domaine expérimental du Magneraud \\ B.P. 52, F 17700 Surgères
}

\begin{abstract}
Résumé
La qualité de la fourrure (poids et surface de la peau, compacité du pelage, longueur du poil) de 381 lapins élevés au Domaine expérimental du Magneraud (I.N.R.A.) est analysée relativement à 4 facteurs de variation : le sexe, le milieu d'élevage après le sevrage - soit dans des cages placées à l'intérieur d'un bâtiment conventionnel, éclairé et non chauffé, soit dans des parcs extérieurs de $200 \mathrm{~m}^{2}$ - la bande - animaux abattus en septembre, décembre, mars et juin - et l'âge à l'abattage qui varie entre 11 et 23 semaines. La mortalité est plus forte dans les parcs extérieurs. Les peaux produites par les mâles sont plus lourdes ainsi que celles qui proviennent d'animaux abattus en automne, en hiver ou au printemps et celles obtenues dans les parcs extérieurs, où nous observons par ailleurs une compacité du pelage plus élevée. Les deux souches produisent des peaux très différentes. L'importance des modifications de la valeur commerciale des peaux dues aux mues, phénomène non pris en compte ici, n'a pas permis de déterminer un âge à l'abattage optimum. Il est nécessaite d’envisager des expériences complémentaires.
\end{abstract}

Mots clés: lapin, qualité de la fourrure, milieu d'élevage, saison.

\section{Introduction}

Au cours de ces dix dernières années, l'élevage du lapin en France a beaucoup évoluć; la progression des techniques a entraîné un accroissement notable de la production pondérale des femelles présentes dans les ateliers rationnels (Hrafaf et al., 1983) mais aussi une dégradation de la qualité des fourrurcs (Thomas, 1977). La disparition des petites unités fermières a provoqué celle des structures commer-

(ce travail a fait lobiet dune action thématique programmée en 1980 dans le cadre des travaux de la commission spécialisée lapin de l'I.N.R.A. Cette action a concerné trois laboratoires de I'I.N.R.A. (Pelages, Toisons et Fourrures à Jouy-en-Josas, Elevage du Lapin et Amélioration Génétique des Animaux à Toulouse), ainsi que l'Ecole Française de Tannerie de Lyon. Elle s'est déroulée sur le domaine expérimental du Magneraud. 
ciales qui assuraient le ramassage des peaux (Delaveau, 1981). Si, il y a encore quelques années, la vente de la peau contribuait à amortir la plus grande partie des frais d'abattage, il n'en est plus de même aujourd'hui (Leonardi-Magdelaine, 1984).

I1 nous a donc paru intéressant d'étudier quelques-uns des facteurs de variation de la qualité d'une peau, pour éventuellement définir ultérieurement une ou plusieurs méthodes d'élevage du lapin permettant d'obtenir des peaux de qualité. Dans l'optique d'une utilisation par des fourreurs, la qualité d'une peau de lapin dépend de plusieurs critères (VRILlon \& Rochambeau, 1980); les principaux sont : la densité ou la compacité du pelage ainsi que la finesse du poil, le poids et la surface de la peau (KASOWSKI, 1957). Nous inspirant de diverses expérimentations réalisées dans des pays d'Europe de l'Est (Kaverin, 1977 et 1979 ; Volchenko, 1975), nous étudions les variations de plusieurs critères mesurés sur les peaux en fonction du sexe, du milieu d'élevage des animaux après le sevrage, de la saison d'abattage, de l'âge de l'abattage et de la souche utilisée. Ce protocole est original dans la mesure où les seuls résultats disponibles à notre connaissance, ont été obtenus dans un contexte (climat, type de bâtiment, régime alimentaire, souche utilisée...) très différent de celui que nous rencontrons; ce protocole devrait permettre d'apprécier ce qui est transportable.

\section{Matériel et méthodes}

\section{A. Matériel animal}

Les reproducteurs sont élevés dans un bâtiment conventionncl. Cet ancien poulailler, isolé et ventilé de façon statique, possède des fenêtres et n'est pas chauffé. Notre expérimentation débute après le sevrage des lapins qui a lieu dans la cinquième semaine d'âge, c'est-à-dire entre le $28^{\prime \prime}$ et le $31^{\circ}$ jour après leur naissance. Nous comparons deux souches, baptisées $\mathrm{N}$ et $\mathrm{R}$.

Les animaux de la souche $\mathrm{N}$ sont issus d'un croisement entre une femelle «hybride» d'une souche commerciale qui a un poids adulte variant entre 3,7 et $4,0 \mathrm{~kg}$ et un mâle albinos d'un format légèrement plus élevé.

Les animaux de la souche $\mathrm{R}$ proviennent des cheptels Rex qui sont élevés au Magneraud; le poids adulte des femelles est le plus souvent compris entre 2,8 et $3,3 \mathrm{~kg}$. Le pelage du lapin possède trois types de poil : les jarres, les barbes et les duvets (Rougeot, 1974); chez les lapins à poil normal, comme ceux de la souche $\mathrm{N}$, les jarres atteignent presque $40 \mathrm{~mm}$ alors que les barbes ne font qu'un peu plus de $20 \mathrm{~mm}$. Chez les lapins Rex, les jarres et les barbes ont une longueur semblable à celle des duvets (Robinson, 1958 ; LiEnhart, 1962). Les animaux de la souche R, contrairement à ceux de la souche $\mathrm{N}$, n'ont pas fait l'objet d'une sélection visant à améliorer leur productivité numérique et pondérale; ainsi les souches de lapins Rex que nous connaissons ont une productivité plus faible que celle des souches à poil normal utilisées dans les élevages de production (Rochambeau \& VRILloN, 1982). 


\section{B. Milieux d'élevage}

Après le sevrage, la moitié des animaux est élevée dans le même bâtiment que les reproducteurs ; ce bâtiment est éclairé de façon à posséder une durée d'éclairement journalier de 16 heures toute l'année. Les animaux sont placés, par groupe de 15 individus de même sexe, dans des cages grillagées d'un mètre carré.

L'autre moitié est introduite dans des parcs extérieurs de $200 \mathrm{~m}^{2}$. Au centre, un petit abri contient la trémie d'alimentation et un abreuvoir à poulet à niveau constant d'une longueur de $1,50 \mathrm{~m}$. Dans chaque parc, des groupes de 12, 24 ou 48 lapins de même sexe sont introduits. Les densités sont donc de 0,06 - 0,12 - 0,24 animal au mètre carré, le nombre de postes d'alimentation de la trémie étant alors respectivement égal à $4 \cdot 4$ et 8 .

Le sevrage a lieu le mercredi de chaque semaine. Nous disposons de six parcs extérieurs, soit deux par densité, ce qui permet de séparer les sexes. La première semaine, nous mettons en place un lapin dans les parcs extérieurs de densité 12, deux lapins dans les parcs extérieurs de densité 24 et quatre lapins dans les parcs extérieurs de densité 48 . Un nombre équivalent d'animaux est placé à l'intérieur dans des cages collectives. Cette opération est répétée pendant douze semaines consécutives de façon à obtenir respectivement 12,24 et 48 animaux introduits dans chaque parc extérieur. Sept semaines après la dernière introduction, tous les animaux sont abattus; leur âge varie donc entre 11 et 23 semaines. Cet ensemble d'animatux abattus le même jour constitue ce que nous appelons une bande ; 4 bandes se sont succédé et elles ont été abattues respectivement en septembre 1980 , décembre 1980, mars 1981 et juin 1981. Les bandes 1 et 3, ainsi que les bandes 2 et 4 , ont séjourné dans les mêmes parcs extérieurs. Par ailleurs, l'ensemble des animaux sevrés la première semaine à l'intérieur ou dans les parcs extérieurs à l'occasion de chacune des quatre bandes constitue ce que nous appelons la première cohorte; cette opération ayant été répétée pendant douze semaines consécutives, nous définissons ainsi douze cohortes qui ont été sevrées à l'intérieur et douze cohortes qui ont été sevrées dans les parcs extérieurs.

Tous les animaux reçoivent un aliment granulé classique du commerce ad libitum; cet aliment contient un minimum de 15 p. 100 de protéines brutes, un maximum de 14 p. 100 de cellulose brute, et un minimum de 1 p. 100 de matières grasses, ainsi qu'un complément minéral vitaminé et un anticoccidien. Les lapins placés dans les parcs extérieurs peuvent consommer la végétation herbacée qui couvre les parcs au début de l'expérimentation.

\section{Variables étudiées}

Les caractères de croissance qui ont été mesurés sont le poids à l'abattage et le poids de carcasse; on connaît aussi le poids individuel au sevrage, la taille de portée au sevrage dont est issu l'animal étudié, ainsi que l'âge auquel il a été abattu.

Les critères de peau retenus sont au nombre de six : le poids de peau humide mesuré lors de l'abattage, le poids de peau tannée, la longueur et la largeur de la 
peau, la longueur du poil et la compacité du pelage. Dans le cadre de cette expérimentation, Rougeot \& Thebault (Laboratoire des Pelages, Toisons et Fourrures, C.N.R.Z., I.N.R.A.) ont mis au point un appareil permettant d'évaluer la compacité du pelage. Le principe de fonctionnement de cet appareil est de presser avec une force constante une bande de poils dans des conditions standardisées (largeur de pressage des poils sur la peau : $5 \mathrm{~mm}$; hauteur du poil pressé : $5 \mathrm{~mm}$; niveau sur la longueur du poil compris entre 5 et $10 \mathrm{~mm}$; mesure effectuée toujours au même endroit : sur la croupe). La compacité du pelage est en relation avec l'épaisscur de cette bande et grâce à un relais électronique, nous mesurons les variations de l'épaisseur pressée, ce qui nous donne une valeur relative permettant de comparer les compacités de plusieurs pelages (la mesure effectuée étant fonction du nombre de poils et de leur diamètre moyen, les comparaisons n'ont de sens que si le diamètre moyen reste fixe, il faudra donc travailler intra type-génétique et pour un stade défini du pelage).

Cette méthode permet d'évaluer indirectement la densité du pelage, c'est-à-dire le nombre de poils par unité de surface, sans avoir à estimer directement ce nombre.

A partir des variables mesurées, on calcule deux critères de peau supplémentaires : la surface de la peau que nous obtenons en multipliant la longueur de la peau par la largeur de cette même peau, et le poids par unité de surface que nous définissons comme le rapport du poids de la peau humide et de la surface de la peau.

Les poids sont exprimés en grammes, la surface en centimètres carrés, l'âge à l'abattage en jours et la longueur du poil en millimètres.

\section{Méthodes d'analyse}

Nous avons tout d'abord étudié la mortalité observée dans chacune des cellules du dispositif expérimental; un test de $\chi^{2}$ a permis de tester la signification des différences observées. Les seuils de rejet de l'hypothèse d'égalité des mortalités observées sont respectivement 5 p. 100 - * et - p. $100-* * *$

Les variances des diverses variables étudiées dans les souches $\mathrm{N}$ et $\mathrm{R}$ ont été comparées à l'aide d'un test de comparaison de deux variances ordonnées.

L'étude des variables quantitatives s'est faite à l'aide de plusieurs modèles d'analyse de variance. D'une part, nous avons utilisé des modèles à effets fixés avec interaction, comme par exemple le modèle suivant :

$$
\mathrm{X}_{\mathrm{ijk} \mathbf{l}}=\mu+\mathrm{s}_{\mathrm{i}}+\mathrm{m}_{\mathrm{j}}+\mathrm{b}_{\mathrm{k}}+\mathrm{d}_{\mathrm{ij}}+\mathrm{e}_{\mathrm{jk}}+\mathrm{f}_{\mathrm{kl}}+\mathrm{R}_{\mathrm{ijkl}}
$$

$\mathrm{X}_{\mathrm{ijki}}$ est la valeur de l'âge de l'abattage faite sur le eième lapin de sexe (i) placé dans le milieu d'élevage $(j)$ de la bande $(k)$;

$\mu$ est la moyenne générale ;

$S_{i}$ est l'effet du sexe (deux niveaux);

$\mathrm{m}_{\mathrm{j}}$ est l'effet du milieu d'élevage (deux niveaux);

$b_{k}$ est l'effet de la bande (quatre niveaux);

$d_{i j}, e_{j k}, f_{k l}$ sont les effets d'interaction de premier ordre entre les facteurs que nous venons de définir;

$\mathrm{R}_{\mathrm{ijkl}}$ est la résiduelle. 
Les conditions supplémentaires utilisées pour lever l'indétermination s'écrivent :

- pour les effets principaux $\underset{i}{\sum} n_{i \infty o \infty} s_{j}=0$

- pour les interactions $\forall j, \sum_{i} n_{i j o} d_{i j}=0$

$$
\forall \mathrm{i}, \underset{\mathrm{j}}{\sum} \mathrm{n}_{\mathrm{ijo}} \mathrm{d}_{\mathrm{ij}}=0
$$

où $n_{i j k}$ est le nombre de lapins de sexe (i) placé dans le milieu d'élevage ( $\mathrm{j}$ ) de la bande $(k), n_{i o o}=\sum_{j k} n_{i j k}, n_{i j o}=\sum_{k} n_{i j k}$. Ce type de modèle a aussi été utilisé pour analyser l'effet sur le poids individuel au sevrage de la souche, du milieu d'élevage, de la bande, du sexe et du devenir de l'animal ( 2 niveaux : animal mort ou éliminé pendant le protocole et animal abattu à la fin du protocole).

D'autre part, nous avons utilisé des modèles à effets fixés avec interaction, dans lesquels deux covariables ont été introduites. Nous avons par exemple :

$$
\begin{aligned}
X_{i j k l}=\mu+s_{i}+m_{j} & +b_{k}+d_{i j}+e_{j k}+f_{k l}+\alpha\left(Y_{i j k l}-y \ldots\right) \\
& +\beta\left(Z_{i j k l}-z \ldots\right)+R_{i j k l}
\end{aligned}
$$

$\mu, s_{j}, m_{j}, b_{k}, d_{i j}, e_{j k}, f_{k 1}$ ont la même signification que précédemment. $\mathrm{Y}_{\mathrm{ijjl}}$ est le poids individuel au sevrage, $\mathrm{y} \ldots$... est un paramètre de centrage qui est égal à la moyenne générale et $\alpha$ est le coefficient de régression de la variable $X_{\mathrm{ijkl}}$ sur le poids individuel au sevrage $; Z_{\mathrm{ijkl}}$ est l'âge à l'abattage, $\mathrm{z} \ldots$ est un paramètre de centrage et $\beta$ est le coefficient de régression de la variahle $Y_{\mathrm{ijki}}$ sur l'âge $\grave{t}$ l'abattage. Les conditions supplémentaires nécessaires pour lever l'indétermination sont semblables à celles utilisées dans le modèle précédent. Ce modèle a été utilisé dans les deux souches pour les variables suivantes : poids vif à l'abattage, poids de carcasse chaude, poids de peau humide, poids de peau tannée, surface de la peau, poids de la peau par unité de surface. longueur du poil, compacité du pelage.

Un modèle similaire a aussi été utilisé en prenant comme covariable le poids individuel au sevrage et le poids vif à l'abattage. Dans ce cas, l'âge à l'abattage a remplacé le poids vif à l'abattage dans la liste des variables analysées.

\section{Résultats}

\section{A. Elfectifs traités et mortalité}

Le tableau 1 contient les effectifs de lapins mis en place (1618) et les effectifs de lapins abattus à la fin du protocole (731). La mortalité observée est élevée (45 p. 100), même s'il faut rappeler que l'âge à l'abattage est compris entre 75 et 169 jours (tabl. 2). Cette mortalité n'est pas la même pour chacune des bandes; elle croît régulièrement dans les parcs extérieurs avec le numéro de la bande $(24,47,67$ et 77 p. 100 respectivement pour les bandes $1,2,3$ et 4). Si la mortalité est plus 
TABleau 1

Effectifs des animaux morts ou éliminés durant le déroulement du protocole (au-dessus de la diagonale) et des animaux mis en place (au-dessous de la diagonale).

Number of rabbits dead or eliminated during the experiment (above crossline) and total number of rabbits (under crossline).

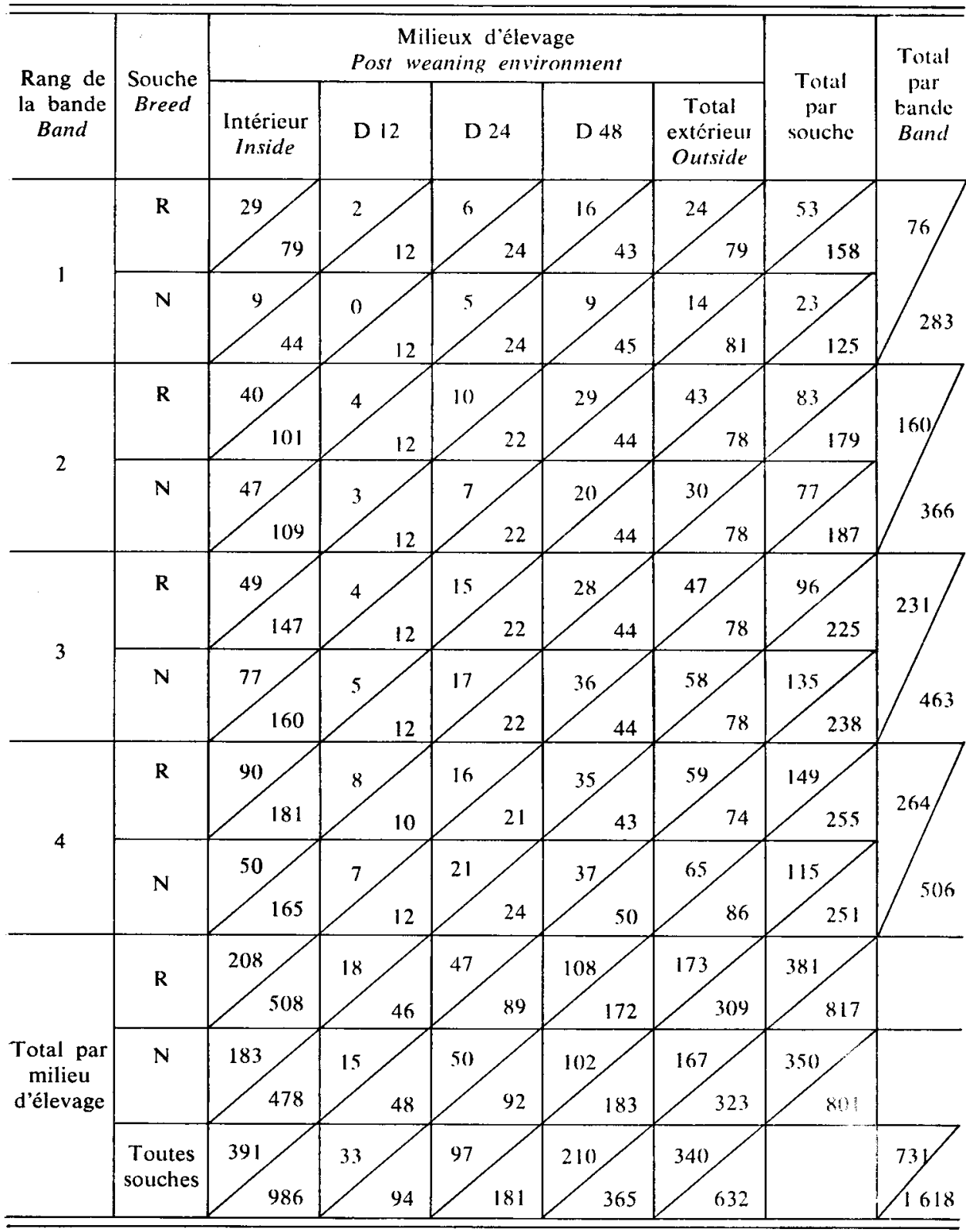




\section{TABleau 2}

Pourcentages de mortalité en fonction de la bande et du milieu d'élevage et signification des différences observées.

Mortality percentages according to band number, raising environment and signification of the observed differences.

\begin{tabular}{|c|c|c|c|c|c|c|c|}
\hline \multirow{3}{*}{$\begin{array}{l}\text { Rang } \\
\text { de la bande } \\
\text { Band }\end{array}$} & \multicolumn{5}{|c|}{$\begin{array}{c}\text { Post weaning environment } \\
\text { Milieu d'élevage }\end{array}$} & \multirow{3}{*}{\multicolumn{2}{|c|}{$\begin{array}{l}\text { Total } \\
\text { par bande } \\
\text { Band }\end{array}$}} \\
\hline & \multirow{2}{*}{$\begin{array}{l}\text { Intérieur } \\
\text { Inside }\end{array}$} & \multirow{2}{*}{$\begin{array}{l}\text { Extérieur } \\
\text { Outside }\end{array}$} & \multicolumn{3}{|c|}{$\begin{array}{l}\text { Densité } \\
\text { Density }\end{array}$} & & \\
\hline & & & D 12 & D 14 & D 48 & & \\
\hline \multirow{2}{*}{1} & 31 & 24 & 8 & 23 & 28 & \multirow{2}{*}{26} & \\
\hline & \multicolumn{2}{|c|}{ NS } & \multicolumn{3}{|c|}{ NS } & & \\
\hline \multirow{2}{*}{2} & 41 & 47 & 29 & 39 & 56 & \multirow{2}{*}{44} & \\
\hline & \multicolumn{2}{|c|}{ NS } & \multicolumn{3}{|c|}{ NS } & & $*$ \\
\hline \multirow{2}{*}{3} & 41 & 67 & 37 & 73 & 73 & \multirow{2}{*}{50} & 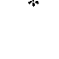 \\
\hline & \multicolumn{2}{|c|}{$(* *)$} & \multicolumn{3}{|c|}{ NS } & & \\
\hline \multirow{2}{*}{4} & 40 & 77 & 68 & 82 & 77 & \multirow{2}{*}{52} & \\
\hline & & & & NS & & & \\
\hline \multirow{2}{*}{$\begin{array}{c}\text { Total } \\
\text { par milieu } \\
\text { d'élevage }\end{array}$} & 40 & 53 & 35 & 54 & 57 & \multirow{2}{*}{\multicolumn{2}{|c|}{45}} \\
\hline & \multicolumn{2}{|c|}{$(* *)$} & \multicolumn{3}{|c|}{$(*)$} & & \\
\hline
\end{tabular}

(*) Au seuil de 5 p. 100.

(**) Au seuil de 1 p. 100.

élevée dans les parcs extérieurs (53 p. 100) qu'à l'intérieur (40 p. 100), cette différence n'apparaît que lors de la réutilisation des parcs extérieurs (bandes 3 et 4). Les différences observées entre les diverses densités dans les parcs extérieurs sont significatives (35, 54 et 57 pour les densités de 12, 24 et 48 lapins par parc). Que ce soit à l'intérieur ou à l'extérieur, la mortalité est la même pour les deux souches (tabl. 3).

De manière à étudier quand cette mortalité intervient, nous avons défini une cohorte comme étant un ensemble d'individus sevrés une même semaine à l'intérieur ou à l'extérieur; en ne tenant pas compte des autres facteurs, on obtient ainsi 12 cohortes qui ont été élevées à l'intérieur et 12 cohortes qui ont été élevées à l'extérieur. Dans les parcs extérieurs (fig. 1), le pourcentage de mortalité cumulé augmente rapidement pendant les six premières semaines et il se stabilise ensuite, 


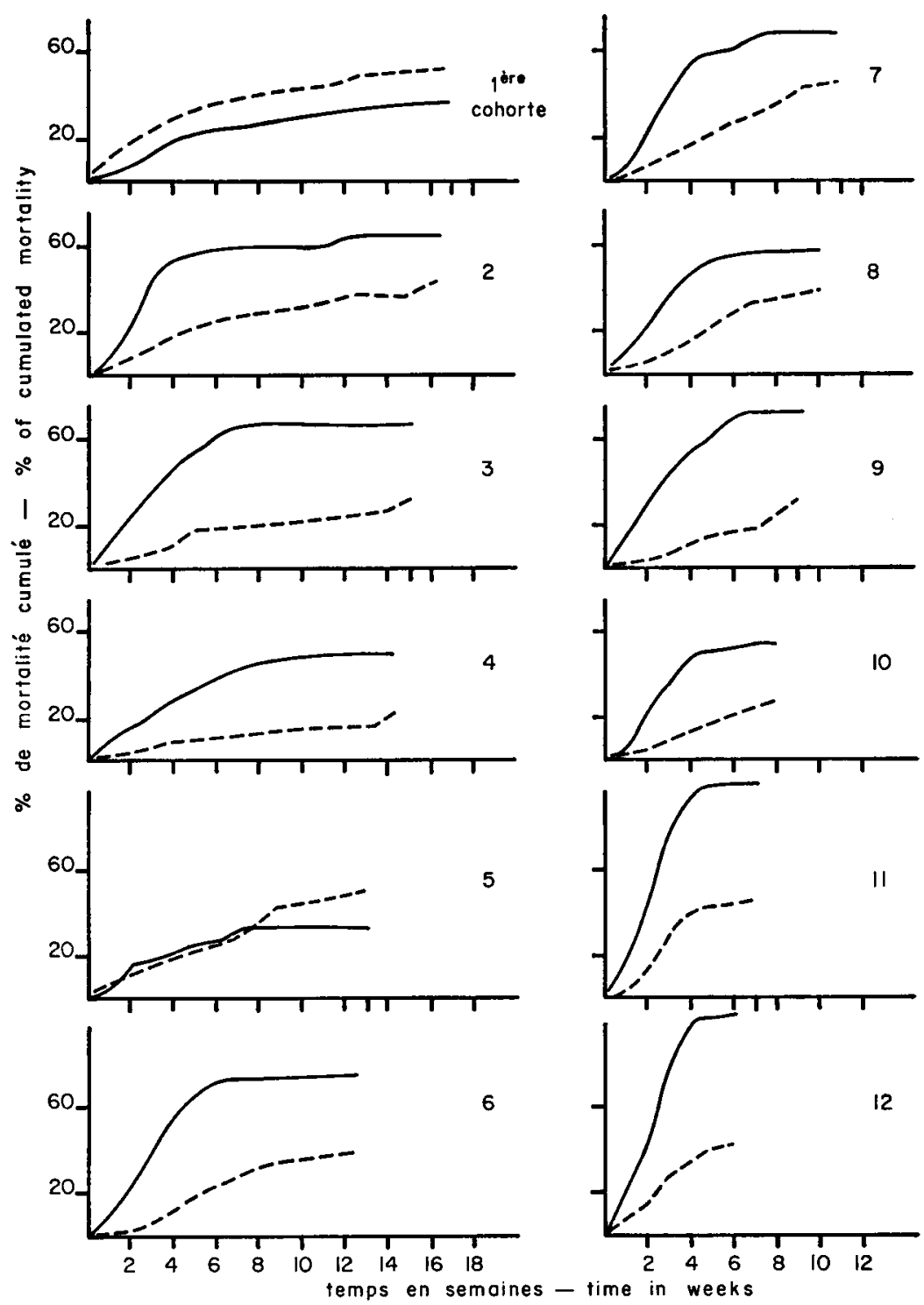

FIG. 1

Evolution du pourcentage de mortalité cumulé en fonction du temps pour différentes durées d'engraissement et pour deux milieux d'élevage (à l'extérieur en trait plein et à l'intérieur en trait pointillé).

Evolution of the percentage of cumulated mortality

in relation with time for various raising periods and for two post weaning environments (outside $=$ full line and inside $=$ dotted line $)$. 


\section{TABLEAU 3}

Pourcentages de mortalité en fonction de la souche et du milieu d'élevage et signification des différences observées.

Mortality percentages according to breed and post weaning raising environment, and significance of the observed differences.

\begin{tabular}{c|c|c}
\hline $\begin{array}{c}\text { Milieu d'élevage } \\
\text { Post weaning } \\
\text { environment }\end{array}$ & \multicolumn{2}{|c}{$\begin{array}{c}\text { Souche } \\
\text { Breed }\end{array}$} \\
\cline { 2 - 3 } $\begin{array}{c}\text { Intérieur } \\
\text { Inside }\end{array}$ & $\mathrm{R}$ & $\mathrm{N}$ \\
\hline $\begin{array}{c}\text { Extérieur } \\
\text { Outside }\end{array}$ & \multicolumn{2}{|c}{$\mathrm{NS}$} \\
\hline $\begin{array}{c}|c| \\
\text { Total par génotype }\end{array}$ & \multicolumn{2}{|c}{$\mathrm{NS}$} \\
\hline
\end{tabular}

(*) Au seuil de 5 p. 100 .

(**) Au seuil de 1 p. 100.

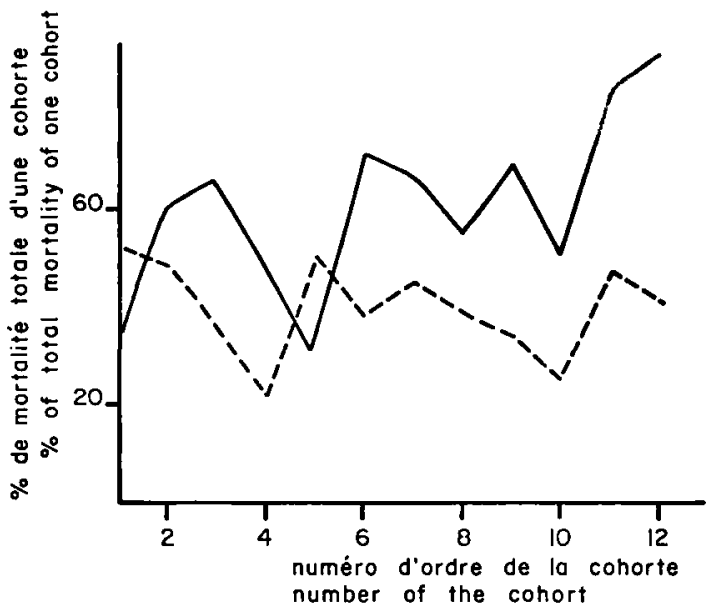

FIG. 2

Evolution du pourcentage de mortalité totale en fonction de la durée d'engraissement pour 2 milieux d'élevage (à l'extérieur en trait plein et à l'intérieur en trait pointillé).

Percentage of total mortality according to raising time for two post weaning environments (outside $=$ full line $;$ inside $=$ dotted line $)$. 
TABLEAU 4

Effectifs des animaux abattus à la fin du protocole dont les fourrures ont été analysées.

Number of slaughtered animals with fur results.

\begin{tabular}{|c|c|c|c|c|c|c|c|c|}
\hline \multirow{2}{*}{$\begin{array}{c}\text { Rang de } \\
\text { la bande } \\
\text { Band }\end{array}$} & \multirow{2}{*}{$\begin{array}{l}\text { Souche } \\
\text { Breed }\end{array}$} & \multirow{2}{*}{$\begin{array}{l}\text { Intérieur } \\
\text { Inside }\end{array}$} & \multicolumn{4}{|c|}{$\begin{array}{c}\text { Milieu d'élevage } \\
\text { Post weaning environment }\end{array}$} & \multirow{2}{*}{$\begin{array}{c}\text { Total } \\
\text { par } \\
\text { souche } \\
\text { Breed }\end{array}$} & \multirow{2}{*}{$\begin{array}{c}\text { Total } \\
\text { par } \\
\text { bande } \\
\text { Band }\end{array}$} \\
\hline & & & D 12 & D 24 & D 48 & $\begin{array}{l}\text { Extérieur } \\
\text { Outside }\end{array}$ & & \\
\hline \multirow{2}{*}{1} & $\mathbf{R}$ & 16 & 5 & 9 & 12 & 26 & 42 & \multirow{2}{*}{82} \\
\hline & $\mathbf{N}$ & 12 & 5 & 6 & 17 & 28 & 40 & \\
\hline \multirow{2}{*}{2} & $\mathbf{R}$ & 42 & 4 & 5 & 10 & 19 & 61 & \multirow[t]{2}{*}{146} \\
\hline & $\mathbf{N}$ & 47 & 5 & 13 & 20 & 38 & 85 & \\
\hline \multirow{2}{*}{3} & $\mathbf{R}$ & 24 & 0 & 0 & 1 & 1 & 25 & \multirow{2}{*}{64} \\
\hline & $\mathbf{N}$ & 29 & 4 & 2 & 4 & 10 & 39 & \\
\hline \multirow{2}{*}{4} & $\mathbf{R}$ & 56 & 1 & 2 & 3 & 6 & 62 & \multirow{2}{*}{89} \\
\hline & $\mathbf{N}$ & 9 & 5 & 3 & 10 & 18 & 27 & \\
\hline \multirow{3}{*}{$\begin{array}{c}\text { Total } \\
\text { par milieu } \\
\text { d'élevage }\end{array}$} & $\mathbf{R}$ & 138 & 10 & 16 & 26 & 52 & 190 & \multirow{2}{*}{381} \\
\hline & $\mathbf{N}$ & 97 & 19 & 24 & 51 & 94 & 191 & \\
\hline & $\begin{array}{l}\text { Toute } \\
\text { souche }\end{array}$ & 235 & 29 & 40 & 77 & 146 & & \\
\hline
\end{tabular}

quelle que soit la durée totale d'engraissement; au contraire, à l'intérieur, le pourcentage de mortalité cumulé augmente régulièrement en fonction du temps (fig. 1). Bien que les animaux des cohortes ayant des numéros d'ordre élevés soient restés moins longtemps dans les parcs extérieurs, la mortalité totale observée sur une cohorte croît avec le numéro d'ordre de la cohorte (fig. 2).

Le nombre d'animaux dont les critères de peau ont pu être mesurés figure au tableau 4 : on voit donc que si 887 lapins ont été abattus, seulement 381 peaux ont pu être analysées. Cette différence importante s'explique par de graves problèmes d'flentification des peaux après le tannage, problèmes qui nous ont conduits à éliminer un grand nombre de données douteuses quant à l'identification. Nous avons vérifié, en analysant le poids vif à l'abattage, le poids de carcasse chaude et l'âge à l'abattage, que l'échantillon sur lequel nous allons travailler est bien représentatif de la population de départ. La faiblesse des effectifs dans certaines cellules élémentaires nous oblige à rassembler les données des parcs extérieurs de densités différentes en un seul milieu d'élevage que nous appelons extérieur. 


\section{B. Analyse des caractères de croissance et des critères de peaux}

La comparaison des variances des 8 variables étudiées dans les souches $R$ et $N$ nous conduit, dans tous les cas, à conserver l'hypothèse d'égalité des variances. Cependant, comme nous l'avons signalé précédemment, les deux souches diffèrent énormément au plan zootechnique et nous allons mener des analyses parallèles dans chacune des souches.

Lors de la mise en place des animaux, un tri a provoqué des différences au niveau des poids individuels moyens au sevrage entre les diverses cellules du dispositif expérimental; pour éliminer ce biais, nous introduisons le poids individuel au sevrage comme covariable.

Par ailleurs, on observe qu'entre les diverses cellules du dispositif expérimental la mortalité totale (tabl. 2 et 3 ), ainsi que la cinétique de cette mortalité diffèrent sensiblement (fig. 1 et 2), ce qui induit des différences significatives sur l'âge à l'abattage (tabl. 5) : les animaux élevés à l'extérieur sont, au moment de l'abattage, plus vieux de 14 jours dans la souche $N$ et de 21 jours dans la souche $R$. Il existe de même un effet de la bande $(-19$ jours et -17 jours pour la première, +6 jours et +1 jour pour la deuxième, +5 jours et +9 jours pour la troisième, +9 jours et +7 jours pour la quatrième, respectivement pour les souches $N$ et $R$ ). Pour éliminer ce biais, nous introduisons l'âge à l'abattage comme covariable. Dans un second temps, nous étudierons l'effet propre de l'âge à l'abattage.

Dans la souche $N$ (tabl. 6), les femelles sont plus lourdes à l'abattage $(+183 \mathrm{~g})$; elles ont des carcasses d'un poids plus élevé $(+102 \mathrm{~g})$ et des peaux plus grandes $\left(+80 \mathrm{~cm}^{2}\right)$, ce qui entraîne une baisse du poids de la peau par unité de surface $\left(-0,029 \mathrm{~g} / \mathrm{cm}^{2}\right)$. A l'extérieur, les animaux sont plus légers à l'abattage $(-271 \mathrm{~g})$, ce qui fournit des carcasses $(-278 \mathrm{~g})$, des peaux humides $(-40 \mathrm{~g})$ et des peaux tannées $(-6 \mathrm{~g})$ moins lourdes. Par contre, la longueur du poil $(+4,1 \mathrm{~mm})$ et la compacité du pelage $(+16,1)$ augmentent. L'effet de la bande est significatif sur le poids vif à l'abattage, le poids de carcasse chaude, le poids de peau humide et de peau tannée, la surface de la peau ainsi que sur son poids par unité de surface. Par rapport à la bande 2 qui peut servir de référence, la bande 1 se singularise par des peaux de grande taille $\left(+129 \mathrm{~cm}^{2}\right)$ relativement plus légères, si on rapporte le poids à une surface $\left(-0,067 \mathrm{~g} / \mathrm{cm}^{2}\right)$; les peaux de la bande 3 sont plus petites (-67 $\mathrm{cm}^{2}$ par rapport à celles de la bande 2 ), caractère qui s'accentue avec la bande $4\left(-107 \mathrm{~cm}^{2}\right)$, ces dernières peaux étant par ailleurs légères dans l'absolu $(-117 \mathrm{~g})$ et relativement à une unité de surface $\left(-0,057 \mathrm{~g} / \mathrm{cm}^{2}\right)$. Il faut signaler que le poids vif à l'abattage des animaux de cette dernière bande était lui aussi plus faible (-356 g). Il n'y a pas d'effet de la bande sur la longueur du poil et sur la compacité du pelage. Il existe sur certaines variables une interaction bandemilieu d'élevage. Le modèle d'analyse de variance, que nous avons utilisé, explique de 26 p. 100 (longueur du poil) à 71 p. 100 (poids de peau tannée) de la variabilité des caractères.

Dans la souche $\mathrm{R}$ (tabl. 7), les résultats observés sont sensiblement différents. Les mâles ont des poids de peaux humides $(+38 \mathrm{~g})$ et de peaux tannées plus élevés $(+18 \mathrm{~g})$, ce qui provoque une augmentation du poids par unité de surface $\left(+0,038 \mathrm{~g} / \mathrm{cm}^{2}\right)$. Les peaux tannées produites à l'extéricur sont plus lourdes 


\section{TABlead 5}

Analyse de covariance de l'âge à l'abattage dans les souches $N$ et $R$, avec 3 effets fixés (sexe, milieu d'élevage, bande) et avec les interactions de premier ordre, le poids individuel au sevrage étant la covariable.

Covariance analysis of slaughter age in breeds $N$ and $R$ with 3 fixed effects (sex, raising environment, band) and 1st order interactions, individual weaning weight being used as a covariable.

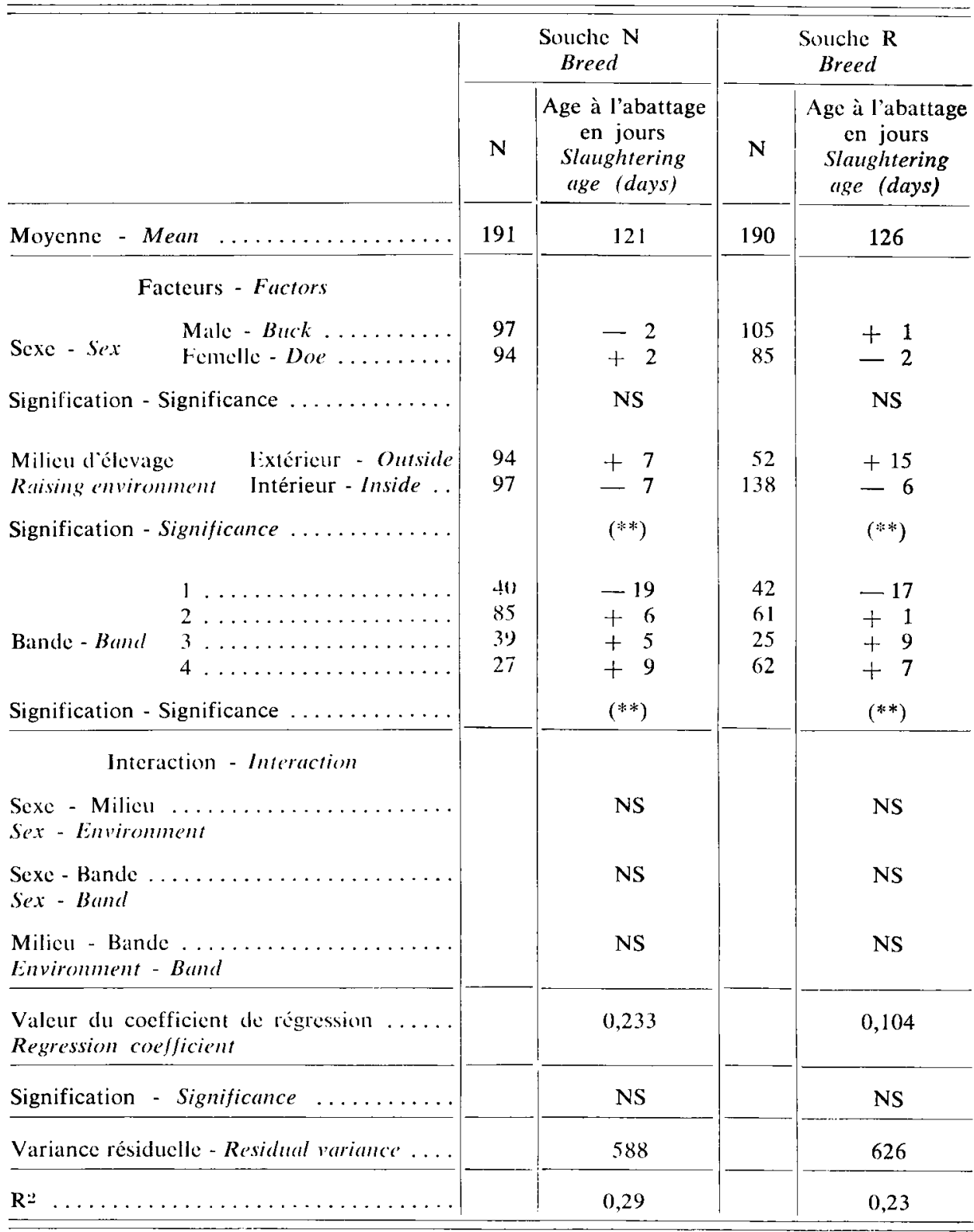


$(+15 \mathrm{~g})$ et la compacité du pelage est plus forte $(+9,5)$. Comme précédemment, il y a un effet de la bande sur l'ensemble des variables sauf la longueur du poil et la densité du pelage. Les variations enregistrées sur la surface de la peau sont plus importantes que celles de la souche $N\left(360 \mathrm{~cm}^{2}\right.$ entre la bande 1 et la bande 4); les autres effets sont assez semblables à ceux que nous venons de décrire. Il existe une interaction bande-milieu d'élevage sur la longueur du poil. Le pourcentage de la variabilité des caractères qui est expliqué par le modèle utilisé est légèrement plus faible que pour la souche $\mathrm{N}$.

Il apparaît qu'entre les diverses cellules du dispositif expérimental il existe des différences sur les poids à l'abattage (effet du sexe, du milieu de la bande dans le cas de la souche $\mathrm{N}$; effet de la bande dans le cas de la souche R). Il nous a donc paru intéressant de voir ce que devenaient les différences que nous venons de décrire en introduisant le poids vif à l'abattage comme covariable à la place de l'âge à l'abattage. Dans la souche N (tabl. 8), l'effet du sexe est modifié : il n'y a plus d'écart significatif entre les poids de carcasse des deux sexes; les mâles ont maintenant des peaux humides $(+31 \mathrm{~g})$ et des peaux tannées $(+18 \mathrm{~g})$ plus lourdes, ce qui augmente la différence sur le poids par unité de surface $\left(+0,037 \mathrm{~g} / \mathrm{cm}^{2}\right)$. De même, les peaux produites à l'extérieur sont maintenant d'un poids plus élevé $(+14 \mathrm{~g}$ pour la peau humide, $+27 \mathrm{~g}$ maximum pour la bande 3 après tannage). Le poids par unité de surface est minimum pour la bande 1 , intermédiaire pour les bandes 2 et 4 . Les autres résultats sont identiques.

Dans la souche $\mathrm{R}$ (tabl. 9), la seule modification notable est celle de l'effet de la bande sur le poids de peau humide : on peut opposer les bandes 1 et 4 qui fournissent des peatix légères ( $-22 \mathrm{~g}$ et $-8 \mathrm{~g}$ respectivement) aux bandes 2 et 3 qui donnent des peaux plus lourdes $(+16 \mathrm{~g}$ et $+18 \mathrm{~g}$ respectivement $)$. Il en résulte que le poids par unité de surface est à nouveau minimum pour la bande 1 , intermédiaire pour la bande 4 et maximum pour les bandes 2 et 3 .

\section{Effet de l'âge à l'abattage}

L'âge à l'abattage est corrélé positivement avec l'ensemble des variables que nous étudions (tabl. 10). Ainsi, dans la souche N, le fort coefficient observé avec le poids vif à l'abattage $(0,72)$ entraîne une forte liaison linéaire avec le poids de peau humide $(0,75)$ et de peau tannéc $(0,79)$. La corrélation est moins forte avec la surface de la peau $(0,50)$ et le poids par unité de surface $(0,48)$; à ce même niveau, on trouve la compacité du pelage $(0,50)$, la longueur du poil se singularisant par une corrélation très faible avec l'âge à l'abattage $(0,28)$. Les résultats se retrouvent dans la souche $\mathrm{R}$, même si l'ensemble des coefficients prend des valeurs légèrement plus faibles. Pour certaines variables, la corrélation est plus forte avec le poids vif à̀ l'abattage.

\section{Discussion}

\section{A. Mortalité observée et déroulement du protocole}

La durée inhabituelle de la phase d'engraissement (de 7 à 19 semaines dans notre protocole au lieu de 7 à 8 semaines dans la majorité des élevages de pro- 


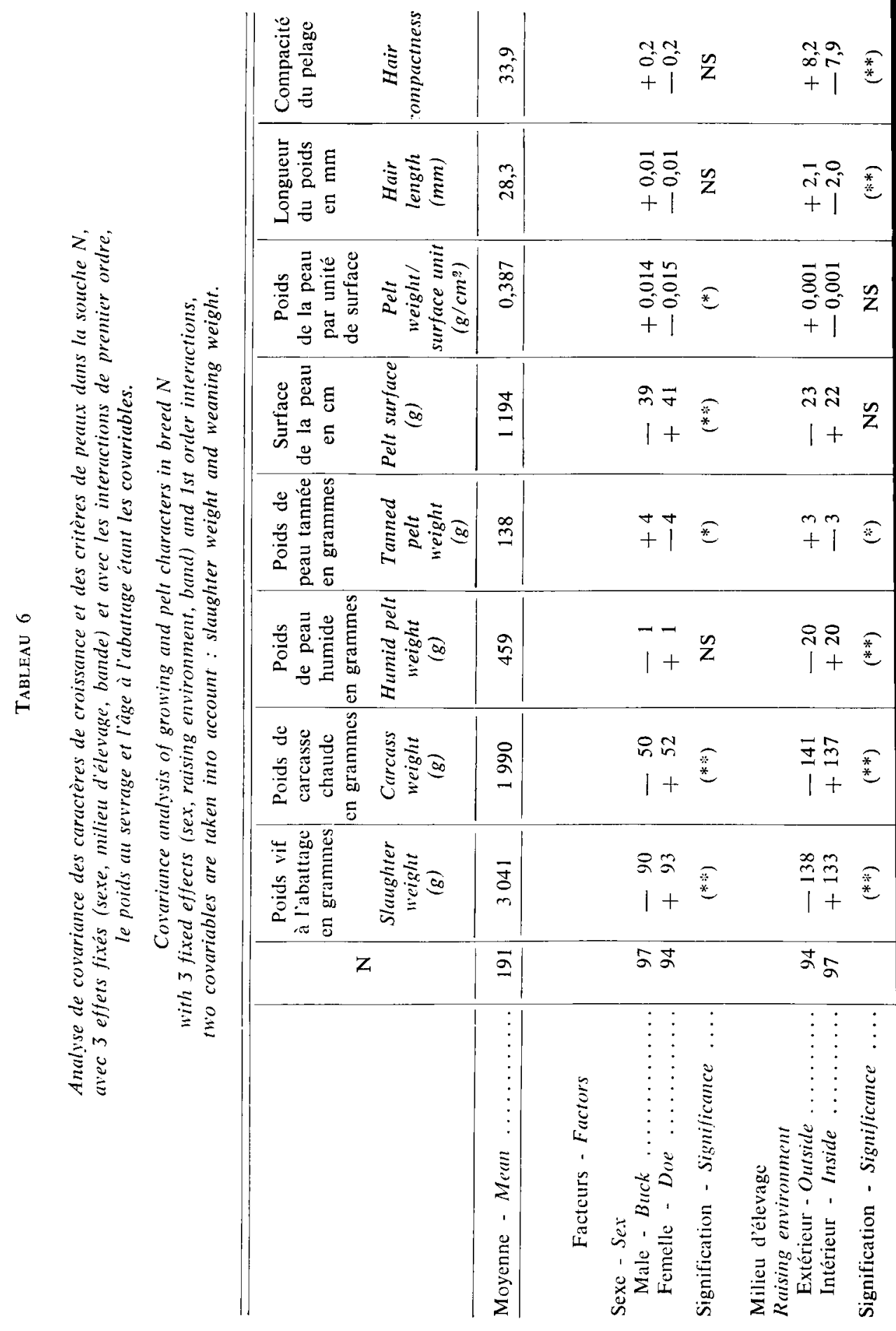




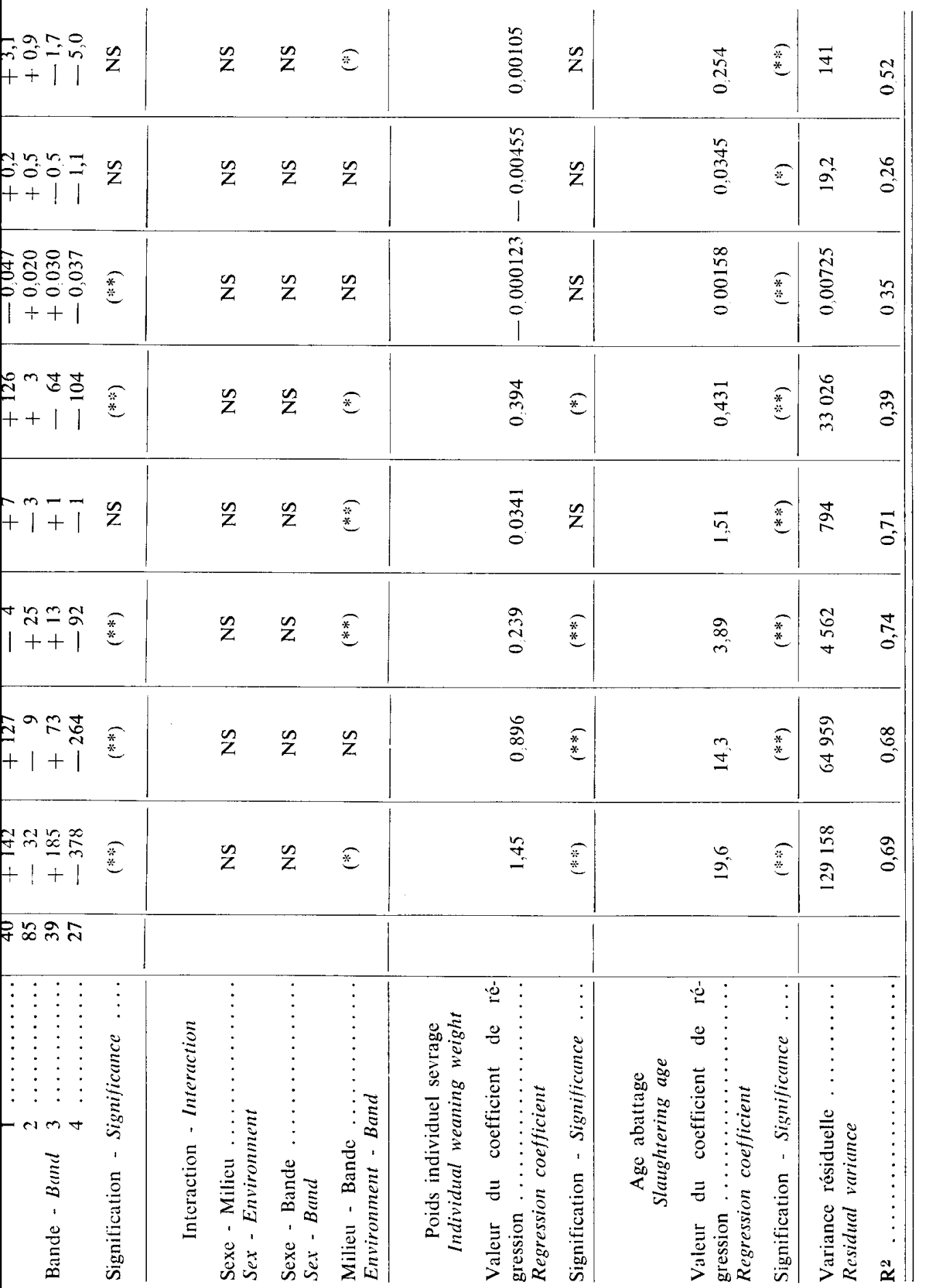




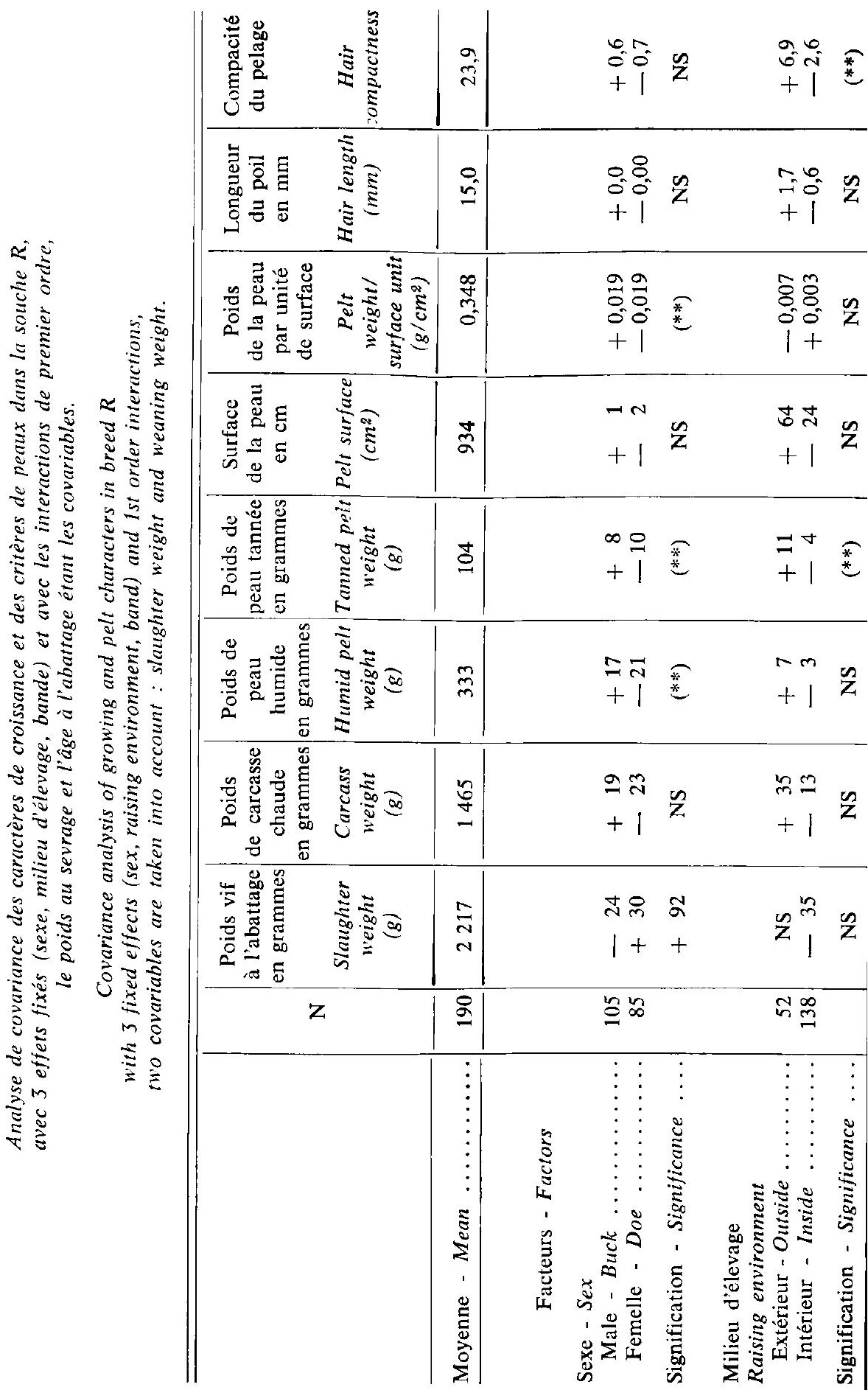




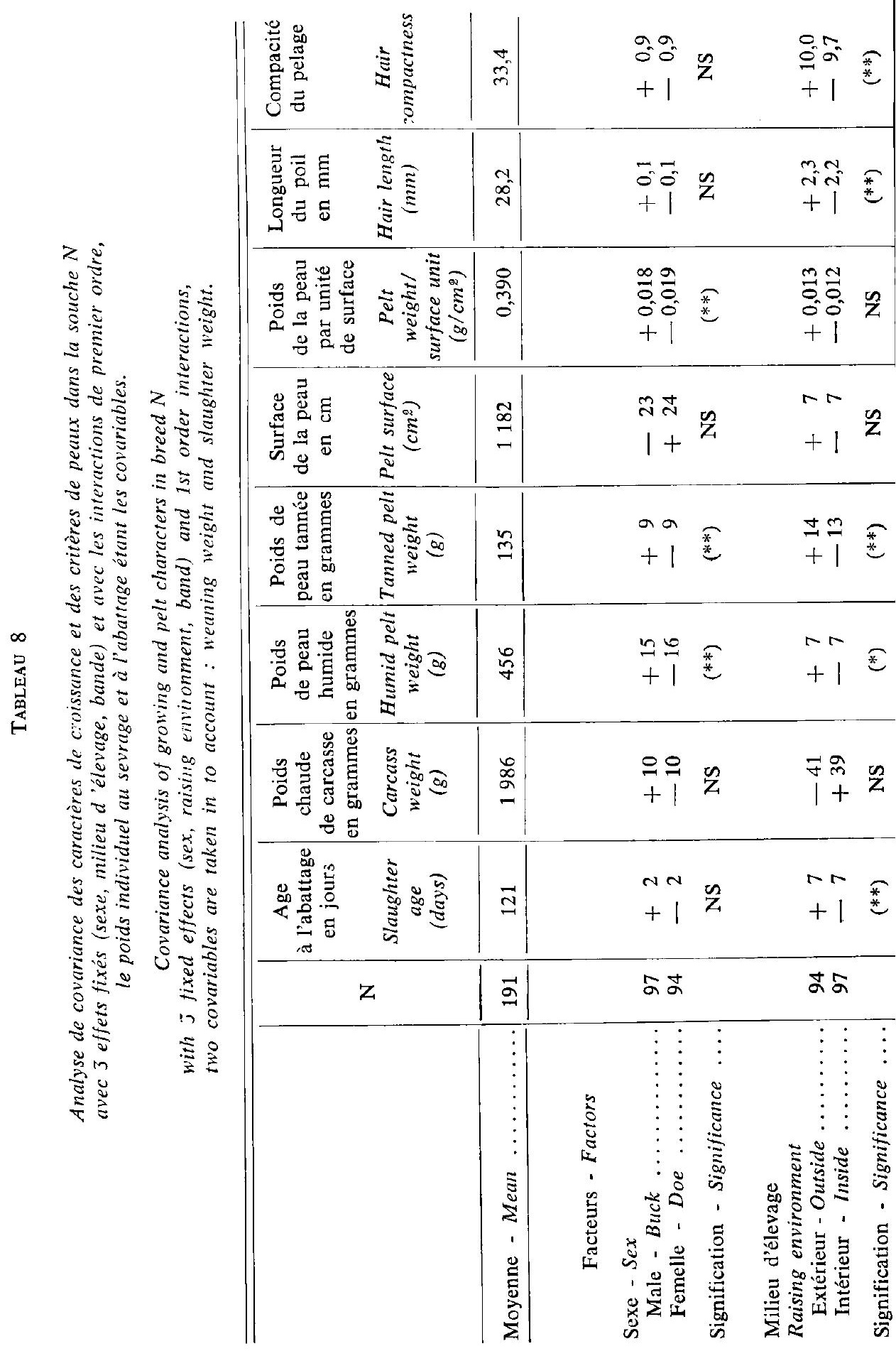




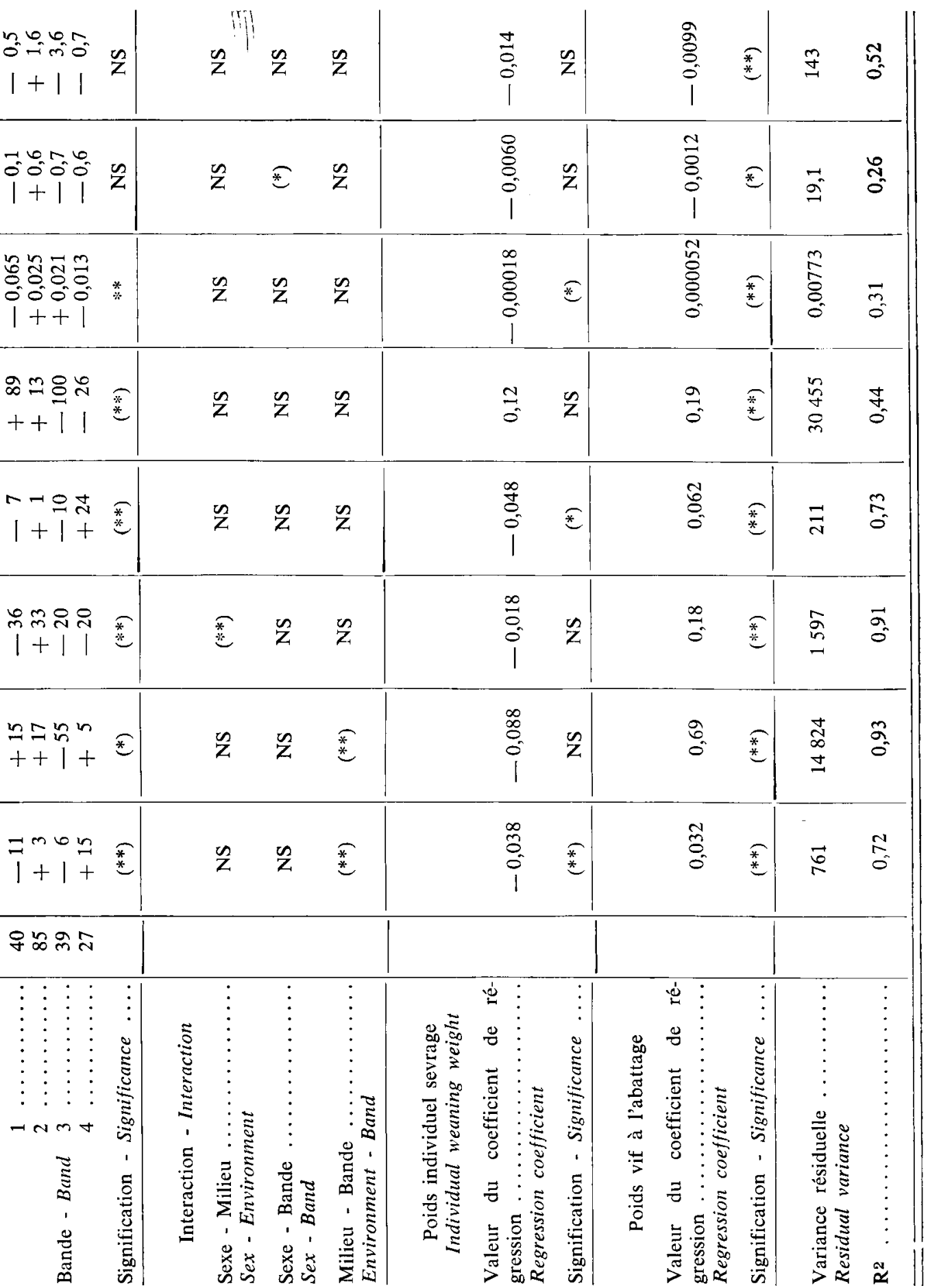




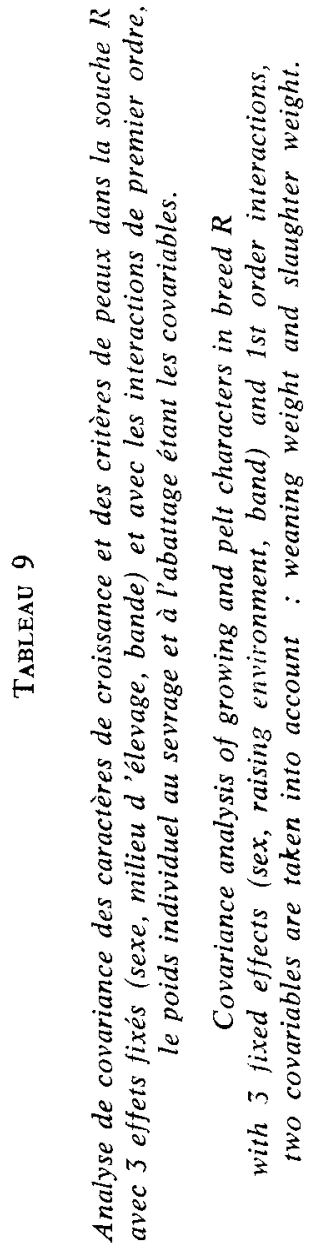

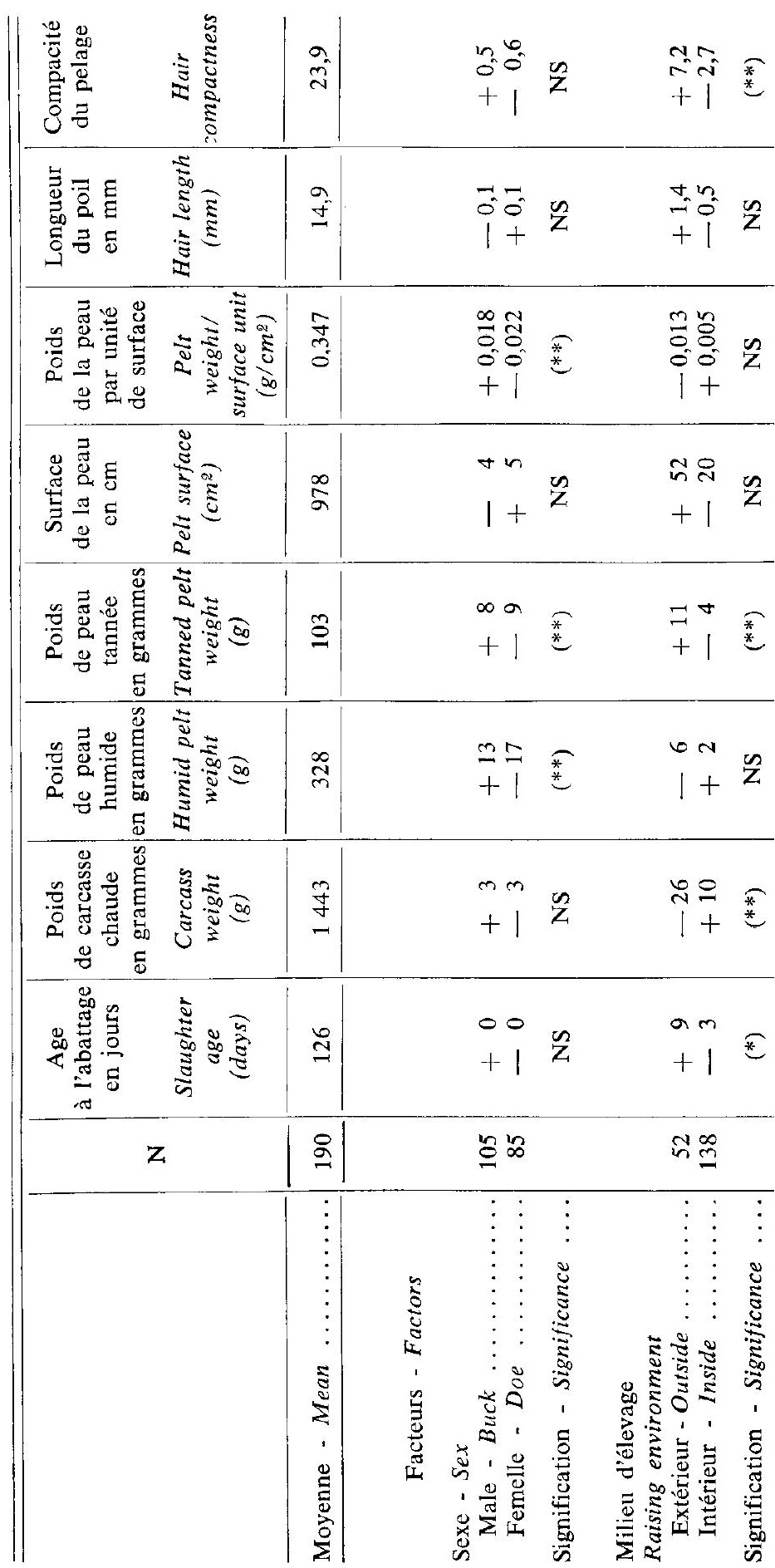




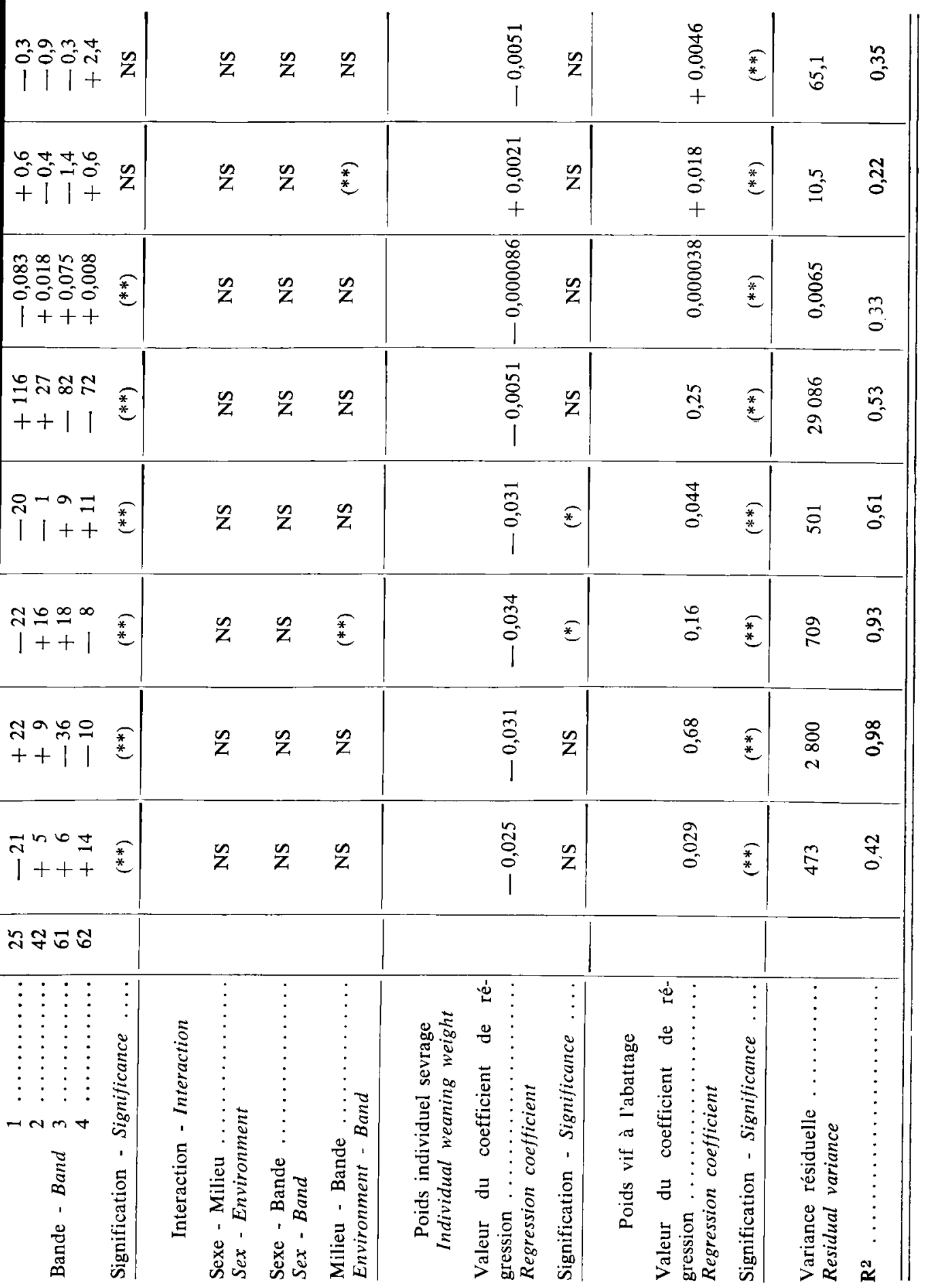




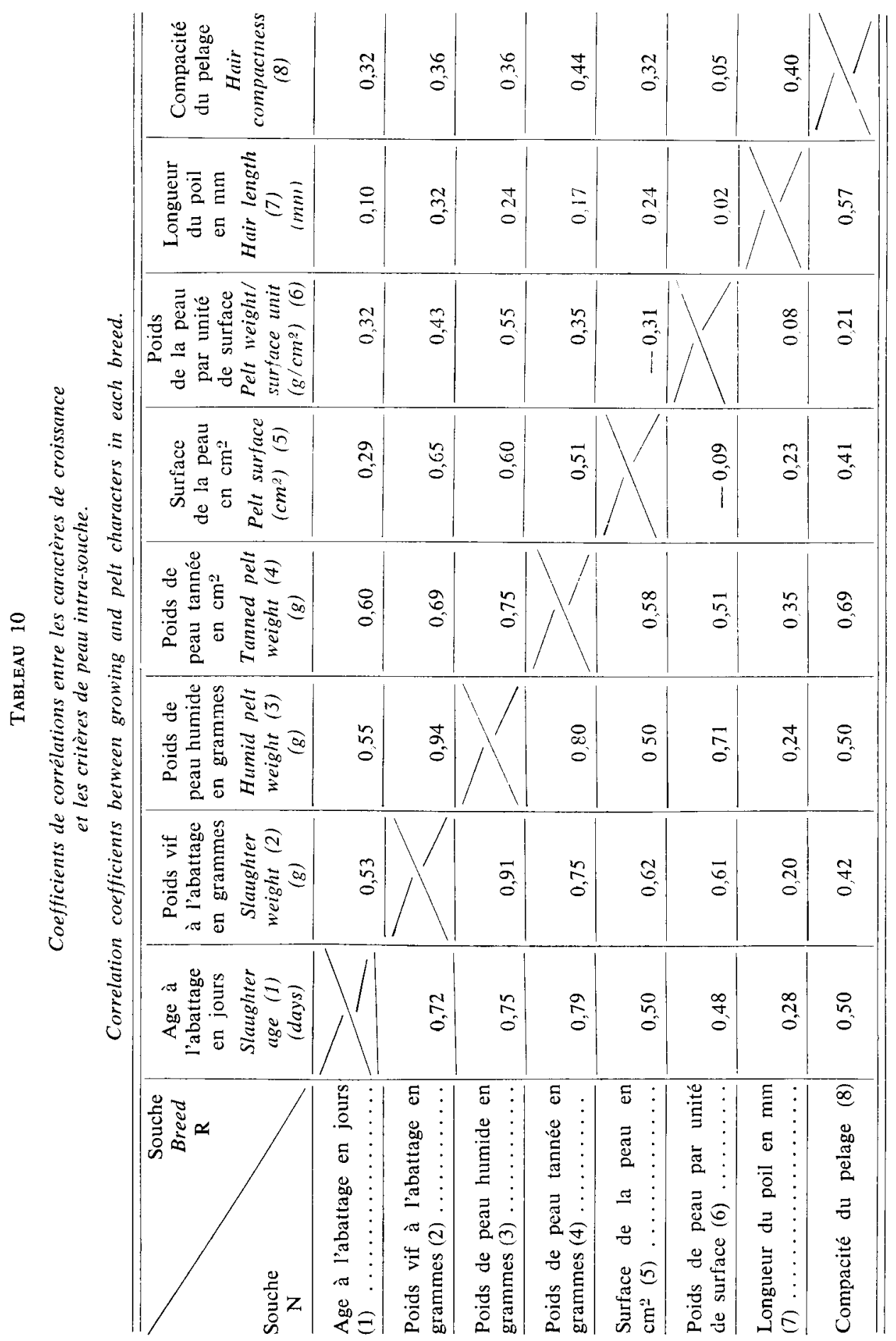


duction) ne suffit pas à expliquer le pourcentage de mortalité observée, puisqu'à l'intérieur, après 6 à 7 semaines, près de $20 \mathrm{p}$. 100 des animaux étaient déjà morts (fig. 1). Nous pouvons donc penser que l'état sanitaire des animaux au moment du sevrage n'était pas bon et que la phase d'engraissement à l'intéricur ne s'est pas déroulée dans de bonnes conditions.

A l'extérieur, plusieurs autres phénomènes sont venus se surajouter. Tout d'abord, les bandes 1 et 3 ainsi que les bandes 2 et 4 ont été élevées dans les mêmes parcs extérieurs; la période de repos n'ayant pas été suffisante entre deux bandes succes. sives, nous avons constaté une infestation parasitaire progressive des parcs. Ainsi, l'effet bande ne peut pas s'interpréter simplement comme un effet saison ; à celui-ci est venu se mêler un effet rang qui n'était pas prévu dans le protocole initial.

Ensuite, l'introduction progressive des animaux dans le dispositif, qui semblait très intéressante sur le plan expérimental, s'est soldée par un échec: la figure 1 montre clairement que les animaux introduits au sevrage dans un groupe déjà constitué d'animaux plus vieux et donc plus lourds sont l'objet d'une mortalité très importante durant les semaines qui suivent le sevrage. Nous pouvons sans doute supposer que leur arrivée provoque une rupture de l'équilibre du groupe, ce qui induit des intolérances entre les animaux et augmente leurs difficultés à s'adapter à ce nouveau milieu.

Enfin, à l'âge de 12 à 14 semaines, les animaux deviennent pubères ce qui, bien 'que nous ayons séparé les sexes, renforce les réactions d'intolérance que nous venons d'évoquer, ce phénomène étant exacerbé par une augmentation de la densité. A ce niveau, il faut constater que la densité la plus faible (12 animaux pour un parc de $200 \mathrm{~m}^{2}$ ) permet de diminuer la mortalité ; cependant, il nous paraît prématuré de condamner les densités plus élevées dans la mesure où, comme nous venons de le voir, plusicurs facteurs sont venus perturber notre protocole.

Ainsi, il ne nous semble pas que la solution des parcs extéricurs soit sans avenir. Il serait intéressant de tester à nouveau cette méthode en constituant une fois pour toutes des groupes d'animaux de même âge, en bon état sanitaire et en conservant une densité faible à modérée (12 ou 24 animaux par parc de $200 \mathrm{~m}^{2}$ ). A l'intérieur, peut-être faudrait-il placer les animaux en cages individuelles après 10 semaines. D'une manière générale, cette mortalité élevée a sans doute eu un effet perturbateur sur l'ensemble de nos résultats, effets qu'il ne nous est pas possible d'apprécier.

Une analyse supplémentaire, non présentée ici, montre que le poids individuel au sevrage des animaux morts ou éliminés avant la fin du protocole et celui des animaux abattus ne diffèrent pas significativement. Nous pouvons donc en déduire que la mortalité n'apparaît pas préférentiellement chez les animaux les plus légers au sevrage, contrairement à ce que nous pensions a priori. Cependant, la validité de cette constatation est réduite par le tri qui a été effectué sur le poids individuel au moment du sevrage.

Comme nous l'avons déjà signalé, la souche $\mathrm{N}$ et la souche $\mathrm{R}$ ne diffèrent pas que par la longueur de leur poil ; la première est issue d'un programme de sélection visant à accroître sa productivité numérique et pondérale, alors que la seconde a seulement été améliorée relativement à un standard. Il n'est donc pas surprenant que les lapins obtenus aient des performances de productivité numérique peu 
comparables (les poids à l'abattage atteignent par exemple $3000 \mathrm{~g}$ pour la souche $\mathrm{N}$ et $2250 \mathrm{~g}$ pour la souche $\mathrm{R}$ ); remarquons cependant que dans les deux cas ces poids à l'abattage représentent un peu moins de $80 \mathrm{p} .100$ du poids adulte de ces souches qui semblent donc être au même degré de maturité (OuHAYoun, 1983). Cette observation nous a conduits à analyser séparément les résultats de chacune des souches. Ce choix nous semble justifié puisque nous venons de voir qu'elles ont répondu différemment au protocole expérimental. Ainsi, la différence entre le poids vif à l'abattage des femelles et des mâles qui existe dans la souche $\mathrm{N}$ conformément à ce que l'on observe classiquement chez le lapin (Rouvier, 1980), n'existe pas dans la souche $\mathbf{R}$; l'apparition plus tardive d'un dimorphisme sexuel pondéral est peut-être un indicateur d'une cinétique de développement différente puisqu'ici le degré de maturité, estimé relativement au poids adulte, nous constatons que la souche R permet d'obtenir à l'extérieur des lapins de même poids vif qu'à l'intérieur. Enfin, la longueur du poil de cette même souche semble être moins variable que celle de la souche $\mathrm{N}$ puisque, par exemple, elle n'est pas sensible à l'effet milieu d'élevage. L'ensemble de ces remarques mériterait d'être confirmé.

Le modèle d'analyse de variance utilisé implique le respect de plusieurs séries d'hypothèses; certaines d'entre elles méritent d'être discutées. Il est en effet douteux que pour les caractères étudiés les variances soient indépendantes de la valeur moyenne du caractère ou du milieu d'élevage; de ce fait, les liaisons entre variables et covariables devraient dépendre des combinaisons de facteurs considérés et nous ne pouvons en aucun cas affirmer que les parts de liaison linéaire considérées dans le modèle sont les mêmes dans chacune des cellules. Une telle situation est inhérente à ce type de comparaison dans laquelle on introduit des systèmes d'élevage modifiant les performances zootechniques. De ce fait, nos conclusions mériteraient d'être vérifiées dans d'autres conditions expérimentales.

\section{B. Intérêt des critères de peau et étude de leurs facteurs de variation}

Les critères de peau que nous avons analysés dans ce protocole sont au nombre de 5 : poids de la peau humide ou tannée, longueur du poil, compacité du pelage, surface de la peau. Ce sont les critères les plus souvent utilisés lorsque l'on cherche à caractériser à l'aide de variables quantitatives la qualité d'une peau dans l'optique d'une utilisation par des fourreurs (KASzOWSKI, 1957; KAWINSKA et al., 1980 ; Volchenko, 1975). La compacité du pelage mesurée sur des peaux tannées (KaszowSKI, 1957) semble être particulièrement intéressante puisqu'elle est corrélée négativement avec l'usure de la peau $(-0,73)$ et positivement avec la force d'arrachement des poils $(+0,52)$; elle est par ailleurs corrélée positivement avec d'autres critères tels que la surface de la peau $(+0,23)$, son épaisseur $(+0,78)$ ou la longueur du poil $(+0,45)$, ainsi qu'avec le poids vif de l'animal $(+0,88)$. Ces valeurs confirment celles que nous observons (tabl. 10). En conclusion, KaszowsKi (1957) indique que les trois critères les plus discriminants qui permettent d'établir une classification commerciale des peaux sont, dans l'ordre, la compacité du pelage, le poids de la fourrure, la surface de la fourrure. Il signale cependant que les peaux ne doivent pas présenter de blessures ou de zones dénudées. Il serait souhaitable de vérifier ces conclusions sur un lot de peaux classées par des professionnels français, analyse qui permettrait en outre de mieux préciser l'intérêt du critère poids de la peau par unité de surface. Si l'on conçoit bien qu'il doit augmenter avec la 
solidité de la peau, il est clair que c'est au dépend de sa souplesse, et qu'il existe donc une borne supérieure à ne pas dépasser.

Avant d'aller plus loin, il convient de revenir sur la mesure de la compacité du pelage : si l'appareil mis au point par Rougeot \& Thebault permet un gain de temps considérable par rapport à un dénombrement exhaustif du nombre de poils sur $1 \mathrm{~cm}^{2}$, le résultat obtenu est sujet à caution. On suppose en effet que le diamètre des poils est constant; or, nous savons que celui-ci varie en fonction de l'âge de l'animal (Rougeot, 1974) et de la saison (Rougeot \& Thebault, 1983). Or, ici nous l'avons utilisé sur des lapins d'âges très différents (11 à 23 semaines) à diverses saisons. Indépendamment d'une poursuite de la mise au point de cet appareil, il faudrait peut-être le réserver à des séries de mesures effectuées au même instant et réalisées sur des lapins d'âge constant appartenant à une seule souche.

Dans ce contexte, il apparaît que les peaux des mâles, qui sont plus lourdes, sont préférables aux peaux des femelles. La différence observée sur les peaux tannées est du même ordre de grandeur que celle qu'indiquent KawinsKa et al. (1980) : $+7 \mathrm{~g}$. Il faut noter que les femelles de la souche $N$ ne compensent pas ce handicap grâce à leur poids vif plus important puisque malgré cela il y a une différence de $6 \mathrm{~g}$ pour une peau tannée par rapport à une peau de mâle. De même, les peaux produites à l'extérieur se distinguent par une compacité du pelage plus élevée. Lorsque ce milieu d'élcvage ne provoque pas une diminution du poids vif à l'abattage (souche $R$ ), les animaux élevés à l'extérieur ont des peaux tannées plus lourdes $(+15 \mathrm{~g})$; nous retrouvons cette différence si on raisonne à poids vif constant dans la souche $\mathrm{N}$ $(+14 \mathrm{~g})$. Ces résultats sont conformes à ceux de KAVERIN (1977 et 1979) qui montre que les peaux obtenues dans des abris en bois se classent mieux commercialement que celles provenant d'animaux élevés dans des bâtiments en brique.

En ce qui concerne l'effet bande, les dates d'abattage indiquent que les animaux de la bande 1 ont été engraissés en été, ceux de la bande 2 en automne, ceux de la bande 3 en hiver et ceux de la bande 4 au printemps. Il faut tout d'abord souligner qu'il n'y a pas d'effet bande sur la compacité du pelage et sur la longueur du poil. Ces résultats sont apparemment contradictoires avec ceux de la bibliographie (RougEOT \& Thebault, 1983) puisqu'on admet que les pelages subissent des variations saisonnières réglées principalement par le photopériodisme, ces variations portant d'une part sur les dimensions des poils (largeur et diamètre) et d'autre part sur le nombre de duvets. Plusieurs causes peuvent masquer cet effet : comme nous l'avons vu, l'effet bande est la résultante d'un effet saison et d'un effet rang et il nous est difficile d'apprécier l'influence de ce dernier effet sur la croissance du pelage. Par ailleurs, les animaux élevés à l'intérieur ont été éclairés 16 heures par jour durant toute la période d'engraissement et n'ont donc pas été exposés aux variations annuelles de la photopériode.

Il y a par contre un effet net de la bande sur le poids et la surface des peaux : celle-ci diminue sensiblement de la bande 1 à la bande 4, alors que le poids de peau humide reste le plus souvent stable pour les 3 premières bandes avant de chuter. Le poids de peau humide par unité de surface permet alors de distinguer 2 types de peaux : celles des bandes 2, 3 et 4 qui sont lourdes et celles de la bande 1 (été) qui sont légères. On obtient le même résultat si l'on raisonne à poids vif constant. Ce classement est aussi celui que donne KAVERIN (1977 et 1979).

Dans les tableaux 6 à 9, il apparaît pour certaines variables des interactions milieu d'élevage-bande qui sont hautement significatives. Une analyse détaillée des résultats 
montre que dans la souche $\mathrm{N}$ la diminution du poids de peau humide et du poids de peau tannée lors de la bande 4 est importante à l'extérieur (par exemple le poids de peau humide n'est plus que de $301 \mathrm{~g}$ contre $422 \mathrm{~g}, 489 \mathrm{~g}$ et $437 \mathrm{~g}$ respectivement pour les bandes 1,2 et 3 ) et plus réduite à l'intérieur (ce même poids de peau humide a pour valeur $485 \mathrm{~g}, 477 \mathrm{~g}, 505 \mathrm{~g}$ et $430 \mathrm{~g}$ respectivement pour les bandes 1, 2, 3 et 4). Dans la souche $R$, l'absence d'effet de la bande sur la longueur du poil pourrait résulter de l'opposition entre les deux milieux d'élevage : en effet, si la longueur du poil ne cesse de croître chez les animaux élevés à l'extérieur avec le numéro d'ordre de la bande $(14,8 \mathrm{~mm}, 15,7 \mathrm{~mm}, 17,5 \mathrm{~mm}, 18,7 \mathrm{~mm}$ respectivement pour les bandes 1,2 , 3 et 4), on observe l'inverse chez les animaux élevés à l'intérieur $(16,4 \mathrm{~mm}, 14,1 \mathrm{~mm}$, $12,8 \mathrm{~mm}, 13,8 \mathrm{~mm}$ respectivement pour les bandes $1,2,3$ et 4 ).

L'influence de l'âge à l'abattage sur la qualité d'une peau a été étudiée par plusieurs auteurs : LAHIARI et al. (1983) et KASzOWSKI (1957) conseillent d'utiliser des animaux le plus vieux et le plus lourd possible; ce dernier auteur donne les valeurs suivantes : poids vif à l'abattage supérieur à $3 \mathrm{~kg}$ et âge à l'abattage compris entre 120 et 130 jours. Ces valeurs sont proches de celles que nous avions choisies. Une augmentation de l'âge à l'abattage permet d'obtenir des peaux plus grandes et plus lourdes, ce qui leur confère une plus grande valeur; simultanément, le poids par unité de surface augmente. Comme nous l'avons déjà signalé, il y a peut-être à ce niveau un seuil à ne pas dépasser.

Sur un plan plus analytique, d'autres auteurs (MiroshnichenKo \& UTKIN, 1972 ; UtKin \& Miroshnichenko, 1972) ont étudié les liaisons entre la compacité du pelage d'une part et le poids et l'âge à l'abattage d'autre part chez des lapins abattus entre 2 et 8 mois, soit à des poids variant entre $1678 \mathrm{~g}$ et $5211 \mathrm{~g}$. Dans ces conditions, ils constatent que la compacité du pelage n'est pas liée linéairement avec les deux variables étudiées. La compacité du pelage mesurée à 2 mois (22 200 poils par $\mathrm{cm}^{2}$ ) est plus forte que celle observée à 4 mois $\left(16000\right.$ poils par $\left.\mathrm{cm}^{2}\right)$ et équivalent à celle obtenue à 8 mois $\left(21500\right.$ poils par $\left.\mathrm{cm}^{2}\right)$. Leurs résultats diffèrent donc sensiblement des nôtres (coefficient de corrélation entre l'âge à l'abattage et la compacité du pelage variant entre 0,32 et 0,50 - tabl. 10), mais ils sont peu comparables car obtenus entre des âges pas trop différents. Ces résultats tempèrent cependant un peu l'intérêt d'une augmentation de l'âge à l'abattage dans la mesure où elle ne provoque pas une augmentation systématique de la compacité du pelage ; par ailleurs, ils nous obligent à tenir compte d'un phénomène important dont nous avons déjà parlé : les mues, et plus généralement la cinétique de développement du pelage (Rougeot, 1974).

\section{Conclusion}

La qualité d'une fourrure peut être quantifiée à l'aide de quelques critères quantitatifs (compacité du pelage, poids et surface de la peau) auxquels ils faut ajouter une appréciation plus qualitative portant sur la coloration (problème qui n'a pas été abordé ici), la tenue du poil ou l'absence de zones dénudées. Parmi les nombreux facteurs de variations de ces divers paramètres, il apparaît que les mâles fournissent des peaux plus lourdes, que les peaux produites à l'extérieur ont un pelage plus compact et sont plus lourdes si nous raisonnons à poids vif constant, et qu'enfin les deux souches que nous avons utilisées donnent des peaux très différentes. 
Si l'analyse du facteur saison montre que les peaux d'été sont plus légères, il importe de l'étudier à nouveau en supprimant certains biais apparus lors du déroulement de notre protocole (apparition d'un effet rang, effet du photopériodisme absent à l'intérieur) et en caractérisant l'état des peaux relativement aux cycles des mues, phénomène qui n'a pas été étudié ici. Pour cette raison, les conclusions que nous avons obtenues concernant la détermination de l'âge à l'abattage sont peu précises ; il faudrait reprendre ce problème en intégrant les cycles de développement du pelage chez le lapin.

\section{Remerciements}

Les auteurs tiennent à remercier F. Lebas, J. Rougeot et B. Poujardieu pour leurs critiques et suggestions lors de la lecture du manuscrit.

\section{Summary}

Variation factors of fur quality and growth characteristics in domestic rabbit

Fur quality (pelt weight and surface, hair length and compactness) of 381 rabbits raised in the experimental centre of «Le Magneraud» (I.N.R.A.) was analysed in relation with 4 variation factors : (i) sex; (ii) post weaning raising environment : inside in a conventional rabbitery, lighted and not heated, outside in enclosures of $200 \mathrm{~m}^{2}$; (iii) band : rabbits slaughtered in September, December, March and June; (iiii) slaughtering age between 11 and 23 weeks. The rabbits came from two breeds : breed $\mathrm{N}$ is a commercial rabbit out of a meat buck and a crossbred doe ; breed $R$ is a Rex rabbit. The mortality was higher outside ( 53 p. 100 vs 40 p. 100) and was the same in the two breeds (tables 2 and 3). Male pelts were heavier as well as pelts from animals slaughtered in autumn, winter and spring or pelts from animals raised outside, where fur compactness was also higher (tables 6 and 7). The two breeds gave different results for pelts and furs. The commercial value of the pelt also depended on moulting and this factor was not taken into account in the present scheme. The optimum slaugthering age was not determined and further experiments are required on that topic.

Key words : rabbit, fur quality, post weaning raising environment, season.

Reçu en mars 1984.

Accepté en septembre 1984.

\section{Références bibliographiques}

Delaveau A., 1981. Le ramassage des peaux de lapins. Ethnozootechnie, 27, 21-35.

Henaff R., Sinquin J.P., Lebas F., 1983. La France cunicole 1982. Cuniculture, 10, 1-9.

Kaszowski S., 1957. Une méthode quantitative d'évaluation des peaux de lapin (en polonais). Roczn. Nauk. rol. B., 71, 469-534.

KAVERIN V.V., 1977. Relation entre la qualité d'une peau de lapin et la saison d'abattage (en russe). Krolikovodstvo i Zverovodstvo, 6, 15-16. 
Karerin V.V., 1979. Effet de l'habitat sur la qualité de la peau chez le lapin Chinchilla (en russe). Zverovodstva i Krolikovodstva, 19, 147-158.

Kawinska J., Niedzwiadek S., Tuczynska J., 1980. Etude de la qualité de la fourrure chez le lapin Argenté Français (en polonais). Roczn. Nauk. Zoot., 7, 157-166.

LahIRI S.S., Sastry V.R.B., Mahajan J.M., 1983. Note on the predictability of pelt characteristics from the weight and age of rabbits. Ind. J. Anim. Sci., 52, 1275-1277.

Leonardi-Magdelaine Pascale, 1984. Le marché du lapin reste soumis à de nombreux aléas. Le Courrier avicole, 838, 39-45.

LienhaRT R., 1962. Mise au point de nos connaissances biologiques actuclles sur le lapin Castorrex. Bull. Soc. Lorr. Sci., 2, 84-102.

Miroshnichenko T.K., Utkin L.G., 1972. Changement de la densité du pelage chez le lapin en fonction de l'âge (en russe). Zverovodstva $i$ Krolikovodstva, 11, 114-118.

Ouhayoun J., 1983. La croissance et le développement du lapin de chair. Cuni-Science, 1, 1-15.

Robinson R., 1958. Genetics studies of the rabbits. Bibliogr. genet., 17, 229-558.

De Rochambeau H., Vrillon J.L., 1982. Quelques paramètres de la productivité numérique dans deux souches de lapins Rex. Premiers résultats. $3^{\text {os }}$ Journées Recherche cunicole en France, ITAVI Ed., communication $n^{\circ} 7$.

Rougeot J., 1974. La formation et la croissance des pelages. In : Thiers H., Cotte J., Actualité de Dermopharmacologie, 6, 1-29. Centre européen de Dermopharmacologie, 33, cours Eugénie, 69003 Lyon.

Rougeot J., Thebault R.G., 1983. Variations saisonnières de la composition et de la structure du pelage : exemple de la toison du lapin Angora. Ann. Zootech., 32, 287-314.

Rouvier R., 1980. Génétique du lapin (Oryctolagus cuniculus). $2^{e}$ Congrès Mondial de Cuniculture, 1, 159-191, Barcelone (Espagne).

Thomas C., 1977. Que deviennent les peaux de lapins? Cuniculture, 4, 86-88.

UtKin L.G., Miroshnichenko T.K., 1972. Relation entre le poids vif et la densité du pelage chez le lapin (en russe). Zverovodstva i Krolikovodstva, 11, 126-133.

Volchenko K.D., 1975. Qualité de la peau chez les lapins Néo-zélandais blancs. Krolikovodstvo $i$ Zverovodstvo, 4, 36-41.

Vrilloon J.L., de Rochambenu H., 1980. Problèmes posés par la production de fourrure de lapin : bibliographic et projet d'étude. 2" Congrès Mondial de Cuniculture, 1, 355-362. Barcelone (Espagne). 